\title{
THE PREDICTIVE VALUE OF SOME RECENT PARAMETERS FOR DIAGNOSIS OF SPONTANEOUS BACTERIAL PERITONITIS
}

\author{
By \\ AIDA MOHAMMED IBRAHIM ${ }^{1}$, NAGLAA GAMAL ABOUL-AZAYM ${ }^{1}$, \\ MEDHAT ALI SALAH ${ }^{1}$, SAYED MOHAMED MOHII-ELDIN ${ }^{2}$, \\ and ABDALLAH MAHMOUD ABDALLAH ${ }^{3}$
}

Departments of Clinical Pathology ${ }^{1}$, Tropical Medicine ${ }^{2}$ and Internal Medicine ${ }^{3}$, Faculty of Medicine, Al Azhar University's Hospitals, Cairo, Egypt

\begin{abstract}
Spontaneous Bacterial Peritonitis (SBP) is a frequent and severe complication in patient with cirrhosis and ascites due to CLD with high mortality rate (20\%-40\%). SBP causes an inflammatory reaction resulting in an increased number of PMNs in ascitic fluid. It was reported that diagnosis of SBP is established when the ascitic fluid PMN count is greater than 250 cells $/ \mathrm{mm} 3$ Lysis of the PMNs during transport to the laboratory, may occur which may lead to false negative results. This study evaluated the usefulness of some recent parameters for diagnosis of SBP that can be used for future development of a rapid bedside test. Identifying a sensitive marker that can be used for rapid diagnosis of SBP in cirrhotic patients will have important clinical and economic consequences for this group of patients and also for clinicians involved in their cares. This study was conducted on 100 patients with ascites admitted to Tropical Medicine department, Al-Azhar University Hospitals in the period from January 2015 to August 2015. The results showed that there was a highly significant positive correlation between ascetic fluid LAF level and neutrophils in ascitic fluid in cases of SBP, Also there was positive correlation between ascetic fluid LAF and ascetic fluid LDH ( $p$ value $<0.001$ ).
\end{abstract}

Key words: Egypt, Spontaneous Bacterial Peritonitis,

\section{Introduction}

Spontaneous bacterial peritonitis (SBP) is a frequent and severe complication of decompensated cirrhosis and ascites (Navasa, 1999). It probably originates with the passage of bacteria from the intestinal lumen to the systemic circulation and then to the ascitic fluid (Llovet et al, 1998). Although the concentration of bacteria in ascitic fluid is low, the inflammatory response, as estimated by the concentration of polymorphonuclear leukocytes and cytokines (tumor necrosis factor $\alpha$ and interleukin-6) in ascitic fluid and blood, is very intense (Navasa et al, 1998). Since its first description in the 1970 s, when the mortality rate exceeded $80 \%$, a significant and substantial improvement in the prognosis of SBP has been noted. In more recent prospective studies the mortality rate was reported to be around $20 \%$. On the other hand the percentage of SBP in hospitalized cirrhotic patients with ascites ranges between $10 \%$ \& 30\%, whereas the prevalence of SBP among outpatients with cirrhosis is markedly lower about $3.5 \%$
(Wallerstedt et al, 2007).

The key pathogenic mechanism that starts SBP is bacterial translocation (BT), a process by which both viable and non-viable enteric bacteria, as well as their products (endotoxins, DNA), cross the intestinal mucosal barrier to infect mesenteric lymph nodes, whence they enter the bloodstream and then ascitic fluid (AF). Patients with a reduced defensive capacity in their AF have been shown to be more susceptible to peritonitis development. All three major defense mechanisms preventing BT in normal subjects are impaired during cirrhosis: intestinal flora stability, intestinal epithelium integrity, and host's immune defense (Wiest, 2011).

A broad range of signs and symptoms are seen in spontaneous bacterial peritonitis (Dănulescu et al, 2015). A high index of suspicion must be maintained when caring for patients with ascites, particularly those with acute clinical deterioration. The completely asymptomatic cases have been reported in as many as $30 \%$ of patients. Fever and chills occur in as many as $80 \%$ of patients. Ab- 
dominal pain or discomfort is found in as many as $70 \%$ of patients. Abdominal tenderness is found in more than $50 \%$ of patients with spontaneous bacterial peritonitis. Findings can range from mild tenderness to overt rebound and guarding. In some cases, the abdominal examination findings mimic an acute intra-abdominal catastrophe requiring emergency surgical evaluation. Physical examination may also disclose hypotension (5-14\% of patients) or signs of hepatic failure such as jaundice and angiomata (Chi et $a l, 2015)$.

The diagnosis of SBP is primarily based on polymorphonuclear (PMN) count in AF as obtained using diagnostic paracentesis. SBP is established when PMN numbers are equal to or greater than $250 / \mu l$ in absence of a surgically amenable intra-abdominal infectious site. In addition to cell counts, AF samples should be always obtained for culture. A positive culture is not necessary for diagnosis given its low sensitivity because of low bacterial levels in AF. Other infection markers such as serum procalcitonin and ascitic lactoferrin have proven useful for diagnosis of SBP, but more studies were needed before its use might be recommended (Runyon, 2013).

The aim of the study was to determine the prevalence of eosinophilic esophagitis in patients with upper gastrointestinal symptoms.

\section{Materials and Methods}

A prospective study involving 100 patients with ascites who were admitted to Tropical Medicine department, Al-Azhar University Hospitals; Al-Hussein and Sayed Galal Hospitals.

The patients were divided into two groups. GI (Patients): included 60 patients suffering from End stage liver disease (ESLD) with ascites secondary to chronic HCV and diagnosed as SBP and GII (Controls): included 40 patients suffering from ESLD with ascites secondary to chronic HCV but without SBP. Generally speaking, diagnosis of peri- tonitis as a complication of cirrhosis is an important clinical problem

Inclusion criteria: Patients who agreed to participate in the study by informed written consent. All patients are affected by decompensated liver cirrhosis and have hepatic ascites and presenting by picture suggesting of SBP like fever, generalized abdominal pain and tenderness.

Exclusion Criteria: These were as follows: 1- Receiving antibiotics during the past week or outside the hospital for any reason; 2- History of abdominal surgery in the past month; 3- Renal dysfunction; 4- Secondary bacterial peritonitis; 5- Peritonitis carcinomatosis; 6- Pancreatic peritonitis; 7- Malignancy, or 8- Tuberculosis.

Diagnosis of SBP was first by eliciting the ascites, then by looking for signs and symptoms consistent with peritoneal irritation, and finally by confirmation with peritoneal fluid testing: 1- Clinical presentation: Ascites, fever, generalized abdominal pain and tenderness, and 2-Laboratory investigation: (PMNL in ascetic fluid $>250 / \mathrm{mL}$ ).

All the patients were subjected to the following: 1- Full medical history and physical examination. The presence of any of fever, abdominal pain, rebound tenderness, absence of bowel sounds and pre-hepatic coma manifestation as lack of concentration in decompensated liver disease increase the susceptibility of SBP. 2- Imaging: a- Chest $\mathrm{x}$-ray, and $\mathrm{b}$ - Abdominal \& Pelvic U/S Scanning of the abdomen and pelvis. 3Laboratory investigations: a- Complete blood count (CBC), b- PT. Normal PT regarded as 12 second and International Normalized Ratio (1NR) Normal INR $<$ I, c- Liver functions tests (total plasma proteins, serum albumin, ALT, AST, total and direct serum bilirubin, \&ALP), d- Kidney function test (serum creatinine, blood urea nitrogen.

Asiatic fluid analysis: 1- Cytological, bacteriological and biochemical examination of ascetic fluid, and 2- Examination of ascetic fluid for LAF (Warrell et al, 2003). 


\section{Results}

The present study was conducted on 100 patients admitted to Al-Hussein and Sayed Galal hospitals the period from January 2015 to August 2015. They were 80 males $(80 \%)$ and 20 females (20\%) and their ages ranged between 32-73years (mean 53.2 \pm SD9.2). GI: 60 patients 45 males and 15 females, and GII: included 40 patients suffering from end stage liver disease without SBP 35 males and 5 females.

Table 1: Pattern of clinical manifestation among group

\begin{tabular}{|l|c|c|c|c|}
\hline Parameter & GI & GII & T-test & P-value \\
\hline No symptoms & $10(16 \%)$ & $26(65 \%)$ & 14.08 & $<0.001^{*}$ \\
\hline Fever & $29(48 \%)$ & $4(10 \%)$ & 8.83 & $0.003^{*}$ \\
\hline Abdominal pain & $28(46 \%)$ & $4(10 \%)$ & 8.06 & $0.005^{*}$ \\
\hline Encephalopathy & $26(43 \%)$ & $4(10 \%)$ & 6.63 & $0.008^{*}$ \\
\hline Jaundice & $12(20 \%)$ & $4(10 \%)$ & 1.01 & 0.265 \\
\hline Splenomegaly & $36(60 \%)$ & $10(25 \%)$ & 11.61 & $0.001^{*}$ \\
\hline
\end{tabular}

Table 2: Comparison between cases and controls as regard liver function tests

\begin{tabular}{|l|c|c|c|}
\hline Parameter & GI $($ mean \pm SD) & G II (mean \pm SD) & P-value \\
\hline Serum albumin (g/dl) & $2.41 \pm 0.45$ & $2.6 \pm 0.4$ & 0.93 \\
\hline Total bilirubin (mg/dl) & $1.7 \pm 0.4$ & $1.5 \pm 0.3$ & 1.1 \\
\hline Direct bilirubin (mg/dl) & $0.6 \pm 0.2$ & $0.5 \pm 0.2$ & 1.1 \\
\hline ALT (U/L) & $30 \pm 19$ & $40 \pm 10$ & 0.98 \\
\hline AST (U/L) & $50 \pm 7$ & $49 \pm 10$ & 0.96 \\
\hline Prothrombin concentration (\%) & $58 \pm 10 \%$ & $57 \pm 12 \%$ & 0.98 \\
\hline
\end{tabular}

Table 3: Parameters among the studied groups

\begin{tabular}{|l|c|c|c|}
\hline Parameter & GI $($ mean \pm SD) & GII $($ mean \pm SD) & P-value \\
\hline Glucose $(\mathrm{mg} / \mathrm{dl})$ & $101.5 \pm 32.8$ & $154.6 \pm 51.3$ & $<0.001^{*}$ \\
\hline Total protein $(\mathrm{g} / \mathrm{dl})$ & $1.64 \pm 0.54$ & $2.41 \pm 0.66$ & $<0.001^{*}$ \\
\hline LDH $(\mathrm{IU} / \mathrm{L})$ & $424.9 \pm 154.8$ & $106.1 \pm 37.3$ & $<0.001^{*}$ \\
\hline WBC count $(/ \mathrm{mm} 3)$ & $4150.7 \pm 1202.3$ & $159.5 \pm 72.4$ & $<0.001^{*}$ \\
\hline (SAAG) $(\mathrm{g} / \mathrm{dl})$ & $2.14 \pm 0.36$ & $2.3 \pm 0.48$ & 0.12 \\
\hline
\end{tabular}

Table 4: Mean of Lactoferrin, LDH, leucocytic count, protein, albumin \&glucose in ascetic fluid and sera of patients \&controls.

\begin{tabular}{|c|c|c|c|}
\hline Items & GI (mean $\pm \mathrm{SD})$ & Group II (mean \pm SD) & $\mathrm{P}$-value \\
\hline Lactoferrin(ng/ml) Ascitic fluid & $4040 \pm 1791$ & $138 \pm 46.9$ & $<0.001$ \\
\hline \multicolumn{4}{|c|}{ LDH(IU/L) } \\
\hline Ascitic fluid & $424.9 \pm 154.8$ & $106.1 \pm 37.3$ & 0.002 \\
\hline Serum & $562.6 \pm 126.2$ & $481.6 \pm 136.8$ & 0.148 \\
\hline $\mathrm{A} / \mathrm{S}$ ratio & $0.67 \pm 0.21$ & $0.23 \pm 0.14$ & $<0.001$ \\
\hline \multicolumn{4}{|c|}{ Leucocytes $(/ \mathrm{mm} 3)$} \\
\hline TLC & $4150.7 \pm 1202.3$ & $159.5 \pm 72.4$ & $<0.001$ \\
\hline PMNs & $3451.2 \pm 1148.2$ & $33.2 \pm 13.9$ & $<0.001$ \\
\hline \multicolumn{4}{|c|}{ Total protein(g/dl) } \\
\hline Ascitic fluid & $1.64 \pm 0.54$ & $2.41 \pm 0.66$ & $<0.001$ \\
\hline Serum & $6.11 \pm 1.03$ & $6.64 \pm 0.92$ & 0.04 \\
\hline $\mathrm{A} / \mathrm{S}$ ratio & 0.27 & $0.43 \pm 0.21$ & 0.009 \\
\hline \multicolumn{4}{|c|}{ Albumin(g/dl) } \\
\hline Ascitic fluid & $3.65 \pm 0.36$ & $5.98 \pm .65$ & 0.002 \\
\hline Serum & $2.41 \pm 0.45$ & $2.6 \pm 0.4$ & 0.047 \\
\hline SAAG & $2.14 \pm 0.36$ & $2.3 \pm 0.48$ & 0.128 \\
\hline \multicolumn{4}{|c|}{ Glucose (mg/dl) } \\
\hline Ascitic fluid & $101.5 \pm 32.8$ & $154.6 \pm 51.3$ & 0.005 \\
\hline Serum & $114.2 \pm 31.9$ & $152.3 \pm 42.6$ & 0.062 \\
\hline $\mathrm{A} / \mathrm{S}$ ratio & $0.9 \pm 0.29$ & $1.1 \pm 0.36$ & 0.048 \\
\hline
\end{tabular}

Table 5: Comparison between culture of ascetic fluid among case and control groups

\begin{tabular}{|l|c|c|c|}
\hline Culture results: & G I & GII & P value \\
\hline Positive results: & $(30 \%)$ & $0(0 \%)$ & $<0.001^{*}$ \\
\hline Negative results: & $42(70 \%)$ & $40(100 \%)$ & \\
\hline
\end{tabular}


Table 6: Mean of Lactoferrin, LDH, PMNs, total protein, albumin and glucose content in culture +ve and/ or -ve ascetic fluid of GI.

\begin{tabular}{|l|c|c|c|}
\hline Parameter & Culture positive & Culture negative & P value \\
\hline Lactoferrin(ng/ml), Ascitic fluid & 3498.6 & 5700.8 & 0.18 \\
\hline LDH(IU/L), Ascitic fluid/Serumratio & 0.59 & 0.71 & 0.058 \\
\hline Leucocytes(mm3), PMNs & 4233.5 & 3115.9 & 0.46 \\
\hline Total protein(g/dl),Ascitic fluid/Serumratio & 0.23 & 0.28 & 0.19 \\
\hline Albumin(g/dl), SAAG & 2.1 & 2.16 & 0.62 \\
\hline Glucose, Ascitic fluid/Serumratio & 1.02 & 0.85 & 0.22 \\
\hline
\end{tabular}

Table 7: Correlation between lactoferrin and age, LDH, PMNs, total protein, SAAG \& glucose content of GI.

\begin{tabular}{|l|c|c|}
\hline Characteristics & Correlation coefficient & P- value \\
\hline Lactoferrin Vs Age & 0.067 & 0.643 \\
\hline Lactoferrin Vs LDH ascetic fluid/serum ratio & 0.790 & $<0.001^{*}$ \\
\hline Lactoferrin Vs PMNs & 0.567 & $<0.001^{*}$ \\
\hline Lactoferrin Vs total protein ascetic fluid/serum ratio & 0.459 & $0.001^{*}$ \\
\hline Lactoferrin Vs SAAG & -0.397 & $0.004^{* *}$ \\
\hline Lactoferrin Vs glucose ascetic fluid/serum ratio & -0.613 & $<0.001^{* *}$ \\
\hline
\end{tabular}

Table 8: Accuracy of Lactoferrin, LDH, PMNs, total protein, SAAG and glucose content in diagnosis of SBP.

\begin{tabular}{|l|l|l|l|l|l|}
\hline Variants & Cut off & Sensitivity & Specificity & +ve Predictive & -ve Predictive \\
\hline Lactoferrin(ng/ml), Ascitic fluid & $\geq 260$ & 100 & 100 & 100 & 100 \\
\hline LDH(IU/L) Ascitic fluid/Serumratio & $\geq 0.45$ & 100 & 100 & 100 & 100 \\
\hline Leucocytes(/mm3) PMNs & $\geq 1186$ & 100 & 100 & 100 & 100 \\
\hline Total protein(g/dl), Ascitic fluid/Serumratio & $\geq 0.35$ & 20 & 50 & 50 & 20 \\
\hline Albumin(g/dl), SAAG & $\geq 2.03$ & 66 & 35 & 72 & 21 \\
\hline Glucose Ascitic fluid/Serumratio & $\leq 0.93$ & 44 & 50 & 69 & 26 \\
\hline
\end{tabular}

\section{Discussion}

Generally, the ascites is a gasttoenterological term for an accumulation of fluid in the peritoneal cavity that exceeds $25 \mathrm{~mL}$. Causes of high SAAG ("transudate") are: aCirrhosis $-81 \%$ (alcoholic in $65 \%$, viral in $10 \%$, cryptogenic in $6 \%$ ), b- Heart failure$3 \%$, c- Hepatic venous occlusion: BuddChiari syndrome or veno-occlusive disease, d- Constrictive pericarditis Kwashiorkor (childhood protein-energy malnutrition). Causes of low SAAG are: a-Cancer (metastasis and primary peritoneal carcinomatosis) $-10 \%$, b- Infection: Tuberculosis $-2 \%$ or spontaneous bacterial peritonitis, c-m Pancreatitis $-1 \%$, d-Serositis, e- Nephrotic syndrome and f- Hereditary angioedema (Branco-Ferreira et al, 1998). Other rare causes: are: aMeigs syndrome, b- Vasculitis, c- Hypothyroidism, d- Renal dialysis, e- Peritoneum mesothelioma, and f- Abdominal tuberculosis

SBP is a frequent and severe complication in patient with cirrhosis and ascites due to CLD with high mortality rate (20\%-40\%). SBP is probably related to several impaired defense mechanisms, such as depressed reticuloendothelial system, phagocytic activity, leucocyte dysfunction, reduced serum complement and low bacterial activity of ascitec fluid (Singal et al, 2014).

Some patients with SBP have symptoms and signs clearly suggestive of peritoneal infection whereas others may be completely asymptomatic or there may he minor symptoms only. Therefore, diagnostic paracentesis is used commonly in cirrhotic patients with ascitis to investigate the presence of SBP (Runyon et al, 2013). Also, SBP causes an inflammatory reaction resulting in an increased number of PMNs in ascitic fluid. It was reported that diagnosis of SBP is established when the ascitic fluid PMN count is greater than 250 cells $/ \mathrm{mm} 3$ (Chi et al, 2015).

Lysis of the PMNs during transport to the laboratory, may occur which may lead to false negative results. As well manual measurement of the ascitic fluid PMN is operator dependant, makes quality control difficult, and can delay the diagnosis. In small clinics without a laboratory, a longer time is re- 
quired, sometimes until the next day. On the other hand, automated cell counters provide a reliable, easier, quicker PMN count and reproducible results within a few minutes; they may also considerably simplify the diagnostic approach and clinical management of SBP (Parsi et al, 2008b).

However, coulter counter findings of the neutrophil count have been shown to be inaccurate for relatively low levels of neutrophils in the ascitic fluid. Therefore, the manual PMN counting method is conventionally preferred. However, a recent study demonstrated that automated cell counts have sufficient sensitivity for diagnosing SBP, thus suggesting that this simple method may be used in place of traditional manual counting (Hirayuki et al, 2014).

PMNL counts in ascitic fluid were not always readily available. A delay in antibiotic therapy entails a high mortality rate. Therefore, considerable effort has been placed in developing a rapid and reliable test for diagnosis of SBP (Chi et al, 2015). Reagents strips for detection of leukocyte esterase have been used to test ascitic fluid and diagnose SBP. The sensitivity and specificity of this method have varied in different studies (Nousbaum et al, 2007).

Lactoferrin is an iron-binding protein found in human mucosal secretions as well as in the specific granules of PMNs and released on activation of the cells, and its presence in body fluids is proportional to the neutrophils (so no need for measurement of serum LAF). Therefore, measurement of ascitic fluid LAF could be clinically useful for detection of SBP in patients with cirrhosis. LAF also has been shown to be remarkably stable and resistant to degradation when left at room temperature for exended periods of time. This property makes this marker attractive for clinical use (Chi et al, 2015).

The present study was conducted to evaluate the value of ascitic fluid LAF in diagnosis of SBP compared to other standard methods for diagnosis and to identify a clinically useful cut-off level that could be used for the future development of a rapid bed-side test. The source LAF of is no need for measurement of serum lactoferrin.

As regard age \& sex, in the present study, there was no statistically significant difference between the studied groups regarding the patient's age and gender. regard symptoms, in the present study, the most frequent presenting clinical features among the SBP group were fever in 29 (48\%) patients, abdominal pain in $28(46 \%)$ patients, hepatic encephalopathy in $26(43 \%)$ patients, splenomegaly in $36(60 \%)$ and jaundice in 12 (20\%) patients. Asymptomatic patients constitute a relatively high percentage which they were 10(16\%). Similar findings were reported (Caruntu and Benea, 2006) they found that fever is the most common manifestation of SBP and approximately $10 \%$ of the patients with SBP were symptomatic. Besides, Chi et al. (2015) estimated that most patients with SBP had symptoms and /or signs clearly suggestive of peritoneal infection, especially abdominal pain, fever and altered gastrointestinal motility. In contrary Wallerstedt et al. (2007) reported 8\% of their 133 cirrhotic patients as being asymptomatic and abdominal pain and tenderness were more common in their patients with SBP.

As regard SAAG ratio: In the present study, there was no statistically significant difference between the studied groups regarding SAAG, as both included patients with ascites due to portal hypertension.

The serum-ascites albumin gradient indirectly measures portal pressure. The albumin concentration of ascitic fluid and serum must be obtained on the same day. The ascitic fluid value is subtracted from the serum value to obtain the gradient.

- If the difference is $>1.1 \mathrm{~g} / \mathrm{dL}$, the patient has portal hypertension, with 97 percent accuracy, or If the difference is $<1.1 \mathrm{~g} / \mathrm{dL}$, portal hypertension is not present.

The patients with a serum-ascites albumin gradient below $1.1 \mathrm{~g} / \mathrm{ld}$. (i.e.: without portal hypertension) rarely develop SBP. An ex- 
ception may occur in patients with the nephrotic syndrome (Runyon et al, 2004).

Regarding the ascetic fluid biochemical examination in the present study, the mean ascetic fluid glucose concentration was statistically significantly decreased in group I patients compared to control group which is statistically significantly increased. Lin et al. (2014) found that the concentration of glucose in the ascitic fluid, under normal conditions, is similar to that in the serum since glucose diffused readily across the membranes. However, ascitic glucose concentration decreases due to consumption by bacteria, white blood cells in the ascitic fluid in case of spontaneous bacterial peritonitis.

The mean ascetic fluid LDH concentration in the present study was statistically highly significantly elevated in (SBP) patients than control group. Runyon (2013) reported that lactate dehydrogenase (LDH) in ascitic fluid is released from PMNs that have lysed. The concentration is increased in SBP, and is even further elevated in secondary bacterial peritonitis. One study, for example, analyzed 22 patients whose ascitic fluid was examined before and then during an episode of SBP. The LDH concentration increased significantly during infection.

The mean ascetic fluid total protein concentration in the present study was statistically highly significantly decreased in SBP group than control group.

This result agreed with Guarner and Soriano (2005) who concluded that low protein concentration in the ascetic fluid has been identified as a risk factor for SBP and these patients are candidates to receive long term prophylaxis to reduce the risk of infections and improve survival. Regarding ascetic fluid cultures in the present study, there is 18 patients $(30 \%)$ were culture positive, and 42 patients $(70 \%)$ were culture negative although they were clinically and laboratory diagnosed as SBP patients. This study also agreed with khalifa et al. (2013) who reported that $70 \%$ of SBP patients had negative bacterial culture. So a positive bacterial cul- ture is obtained in the minority of the patients with SBP and results are delayed for several days.

The present study showed that the majority of isolates were E. coli $(66 \%)$. This result went with Park et al. (2003) who reported that Gram negative organisms accounted for over than $60 \%$ of cases of SBP with $E$. coli accounts for nearly half of all cases of SBP followed by Klbsiella species and other Gram negative bacteria. Almost $25 \%$ of cases are caused by Gram positive organisms, with Streptococcal species being the most common.

However Navasa (2008) noted that the rate of infection with Gram positive bacteria is markedly increased represented by Staphylococci group 23\% and Streptococcus9\% and Pneumococcus isolates were $12 \%$ due to the invasive procedures, while the least isolated organisms were Pseudomonas species and Enterobacter species.

In the present study, there was a highly significant positive correlation between ascetic fluid LAF level and neutrophils in ascitic fluid in cases of SBP because LAF is one of the component of neutrophils and as neutrophil destruction usually occurs, this could explain the elevated LAF and positive correlation with WBCs. Also, in the present study there was positive correlation between ascetic fluid LAF and ascetic fluid LDH, as both LDH and LAF are released from disintegrating ascetic fluid neutrophils and their concentrations increase if the PMN count rises highly enough.

However, there was no correlation between LAF and other chemical parameters of ascetic fluid like glucose and protein, although all these parameters are very important keys for diagnosis of SBP and are the main laboratory parameters for diagnosis of SBP in the present study. Moreover all these chemical parameters showed a significantly statistically difference between cases and controls. This study showed that ascetic fluid LAF could discriminate between SBP and non-SBP samples. The highest com- 
bined sensitivity and specificity of the ascitic fluid LAF to detect SBP was achieved at the level of $260 \mathrm{ng} / \mathrm{mL}$.

Parsi et al. (2008a) reported that the cut off value above $242 \mathrm{ng} / \mathrm{mL}$ that had a sensitivity of $95.5 \%$ and specificity of $97 \%$ as well as there was a very significant difference between the LAF cases and controls in this study. In addition, the result of this study agreed with the report of Parsi et aI. (2008b). This indicated that LAF in ascetic fluid proved a very useful marker for the diagnosis of SBP.

On the other hand, Abdel-Razik et al. (2015) stated that spontaneous bacterial peritonitis (SBP) was the important cause of mortality and morbidity in the Egyptian cirrhotic patients with ascites. Hassan and Abdel Rehim (2015) reported that despite intensive management, spontaneous bacterial peritonitis (SBP) was always associated with poor prognosis especially in the hospitalized patients. They concluded that the age, serum creatinine, bilirubin, and sodium were associated with SBP-related in-hospital mortality. The incorporation of these variables into $\mathrm{CP}$ and MELD significantly improves their predictive ability. iMELD followed by CrCTP provided useful prognostic information for critically ill patients with SBP.

As to the treatment, primary prevention of the spontaneous bacterial peritonitis (SBP) is an important strategy to reduce morbidity and mortality in the Egyptian cirrhotic patients with ascites. Elfert et al. (2016) found that Rifaximin was more effective than norfloxacin in the secondary prevention of SBP. The encephalopathy-related mortality and side effects were fewer in the rifaximin group. Salman et al. (2016) found that terlipressin and low-dose albumin plus terlipressin could be used as a therapeutic alternative to standard-dose albumin in high-risk SBP patients. Assem et al. (2016) reported that alternating norfloxacin- and rifaximin-based primary prophylaxis for SBP showed higher efficacy with the same safety profile when compared with monotherapy of norfloxacin.

\section{Conclusion}

Spontaneous bacterial peritonitis (SBP) is an acute bacterial infection of ascitic fluid. Generally, no source of the infecting agent is easily identifiable, but contamination of dialysate can cause the condition among those receiving peritoneal dialysis (PD).

The SBP occurs in both sexes in the children and adults and is a well-known and ominous complication in patients with cirrhosis. Of patients with cirrhosis who have spontaneous bacterial peritonitis, $70 \%$ were Child-Pugh class $\mathrm{C}$, and the development of spontaneous bacterial peritonitis is associated with a poor long-term prognosis.

\section{References}

Abdel-Razik, A, Mousa, N, Elbaz, S, Eissa, M, Elhelaly, R, et al, 2015: Diagnostic utility of interferon gamma-induced protein $10 \mathrm{kDa}$ in spontaneous bacterial peritonitis: single-center study. Eur. J. Gastroenterol. Hepatol. 27, 9: 1087-93.

Assem, M, Elsabaawy, M, Abdelrashed, M, Elemam, S, Khodeer, S, et al, 2016: Efficacy and safety of alternating norfloxacin and rifaximin as primary prophylaxis for spontaneous bacterial peritonitis in cirrhotic ascites: a prospective randomized open-label comparative multicenter study. Hepatol. Int. 10, 2:377-85.

Branco-Ferreira, M, Pedro, E, Barbosa, MA, Carlos, AG, 1998: Ascites in hereditary angioedema. Allergy 53, 5:543-5.

Caruntu, F, Benea, L, 2006: SBP: pathogenesis, diagnosis, treatment. J. Gastroinestin. Liver Dis. 15, I:51-6.

Chi-Huan, Wu, Tsung-Hsing, Chen, ChunYen, Lin, Cheng-Hsun, Chiu, Ming-Yao Su, Cheng-Tang Chiu, 2015: Analysis of ascitic fluid lactoferrin levels in the diagnosis of spontaneous bacterial peritonitis after systemic antibiotic treatment. Adv. Digest. Med. 2:123e127.

Dănulescu, RM, Stanciu, C, Trifan, A, 2015: Evaluation of prognostic factors in decompensated liver cirrhosis with ascites and spontaneous bacterial peritonitis. Rev. Med. Chir. Soc. Med. Nat. Iasi. 119, 4:1018-24.

Elfert, A, Abo Ali, L, Soliman, S, Ibrahim, S, Abd-Elsalam, S, 2016: Randomized-controlled trial of rifaximin versus norfloxacin for secondary prophylaxis of spontaneous bacterial perito- 
nitis. Eur. J. Gastroenterol. Hepatol. 28, 12: 1450-4.

Guarner, C, Soriano, G, 2005: BT and its consequences in patients with cirrhosis. Eur J. Gastroenterol. Hepatol. 17:27-31.

Hassan, EA, Abdel Rehim, AS, 2015: Creatinine modified Child-Turcotte-Pugh and integrated model of end-stage liver disease scores as predictors of spontaneous bacterial peritonitisrelated in-hospital mortality: Applicable or not. J. Gastroenterol. Hepatol. 30, 7:1205-10.

Hirayuki Enomoto, Shinichi Inoue, Akio, Matsuhisa, Shuhei, Nishiguchi, 2014: Diagnosis of spontaneous bacterial peritonitis and in situ hybridization approach to detect an unidentified pathogen. Int. J. Hepatol. Article; ID 634617, 7 pages.

Khalifa, NA, Abdel-Azzez, HA, Hassaneen, A M, El-Shami, EA, Hassanin, HM, 2013: Ascitic fluid lactoferrin as a diagnostic marker for spontaneous bacterial peritonitis. Afro-Egypt J. Infect. Endem. Dis. 3, 2: 49-55.

Lin-Lin, Huang, Harry, Hua-Xiang, Xia, SenLin, Zhu, 2014: Ascitic Fluid Analysis in the Differential Diagnosis of Ascites: Focus on Cirrhotic Ascites. J. Clin. Transl. Hepatol. 2, 1:5864.

Llovet, JM, Bartoli, R, March, F, et al, 1998: Translocated intestinal bacteria cause spontaneous bacterial peritonitis in cirrhotic rats: molecular epidemiologic evidence. J. Hepatol. 28:30713.

Navasa, M, 1999: Treatment and prophylaxis of spontaneous bacterial peritonitis. In: Arroyo V, Ginès P, Rodés J, Schrier RW, eds. Ascites and renal dysfunction in liver disease: pathogenesis, diagnosis, and treatment. Malden, Mass.: Blackwell Science.

Navasa, M, 2008: Treatment and Prophylaxis of SBP in Arroyo V, Gines pigs. Blackwell Science.

Navasa, M, Follo, A, Filella, X, et al, 1998: Tumor necrosis factor and interleukin-6 in spon- taneous bacterial peritonitis in cirrhosis: relationship with the development of renal impairment and mortality. Hepatology 27:1227-32.

Nousbaum, J, Cadranel, J, Nahon, P, et al, 2007: Diagnostic accuracy of the Multistix 8 SG Reagent Strip in diagnosis of SBP Hepatol. 45:1275-81.

Parsi M, Atreja A, Zein N, et al, 2008a: SBP: recent data on incidence and treatment. Cleve Clin. J. Med. 61:695-65.

Parsi, MA, Saadeh, SN, Zein, NN, Davis, GL, Lopez, R, et al, 2008b: Ascitic Fluid lactoferrin for diagnosis of spontaneous bacterial peritonitis. Gastroenterology 135:803-7.

Runyon, B, 2004: Management of adult patients with ascites due to cirrhosis. Hepatol. 39, 3:34

Runyon, BA, 2013: Introduction to the revised American Association for the Study of Liver Diseases Practice Guideline management of the adult patients with ascites due to cirrhosis. Hepatol. 57:1651-3.

Salman, TA, Edrees, AM, El-Said, HH, El-Abd, OL, El-Azab, GI, 2016: Effect of different therapeutic modalities on systemic, renal, and hepatic hemodynamics and short-term outcomes in cirrhotic patients with spontaneous bacterial peritonitis. Eur. J. Gastroenterol. Hepatol. 28, 7: 777-85.

Singal, AK, Salameh, H, Kamath, PS, 2014: Prevalence and in-hospital mortality trends of infections among patients with cirrhosis: a nationwide study of hospitalised patients in the United States. Aliment. Pharmacol. Ther. 40: 105-12.

Wallerstedt, H, Alexander, KC, Leung AC, et al, 2007: Rapid screening tests for diagnosis SBP. Hepatol. 9:185-9.

Warrell, DA, Cox, TN, Firth, JD, Benz, ED, 2003: Oxford textbook of medicine. Oxford: Oxford University Press.

Wiest, R, Lawson, M, Geuking. M, 2014: Pathological bacterial translocation in liver cirrhosis. J. Hepatol. 60:197-209. 